\title{
Development of a High Performance DC Fuse for Electric Vehicle Application
}

\author{
Miki Kitajima*, Shigeyuki Minami*, K. Matsumoto**
}

\begin{abstract}
This paper reports the production process and the features of a newly developed high-performance DC electric fuse to be used for high voltage, high energy system applications, such as electric vehicles, factory inverter circuits, fuel cell systems. The voltage of power circuits used in electric vehicles has increased to $500 \mathrm{~V}$ or more. This shift to higher voltages is done to reduce current, harness weight and improve motor-controller's efficiency. The fuses that protect these circuits must shut out DC arcs, yet their performance needs improving. This paper reports the performance of a new fuse formed by a thin film of Titanium Hydride on copper film. This new fuse has shown great potential in protecting high voltage circuits of future electric vehicles as well as hybrid electric vehicles, and other high voltage DC electric power devices.
\end{abstract}

Keywords: Electric vehicle fuse, Arc discharge, Titanium Hydride, Thermal Spray, Manufacturing method

\section{INTRODUCTION}

It is hard to break high voltage DC arcing. Because of this, fuses made with conventional $\mathrm{SiO} 2$ powder are unreliable for the high voltage $(500 \mathrm{~V}$ or more) used for electric vehicles, etc. To protect electric vehicle harnesses and electronic circuits such as inverters, $1 \mathrm{~ms}$ arc quenching time is required and development is now an urgent issue. Recent developed electric contactor for the main circuiting has the performance of $3000 \mathrm{~A}$ shutdown for $1 \mathrm{~ms}$. For the protection of semiconductor, such as IGBT failure, $1 \mathrm{~ms}$ shutdown-time is essential. The protection to a short circuited semiconductor helps the second failure of harness burn-out as an active safety. So $1 \mathrm{~ms}$ shut-off time is targeted to the fuse development. We already developed a new type of fuse using Metal alloy Hydride knowing that Hydrogen is effective in breaking arcs [Minami et al., 2003]. Because a low temperature activated alloy Hydride is used for the fuse, safety and productivity are difficult issues. The low temperature alloy Hydride fires when it is exposed in the atmosphere. If the fuse container is broken, the alloy will be meltdown because the alloy is exposed in the air. It is more dangerous if Hydrogen gas is simply stored in a fragile fuse container because the fuse container will be often broken during the arcing. This is the reason why simple $\mathrm{H} 2$ gas sealed type is not al all used. Simple H2 gas sealed type is as recently used for DC contactor. While Titanium Hydride releases Hydrogen at about $600{ }^{\circ} \mathrm{C}$ and can be

* Department of Electrical Engineering, Osaka City University, Sumiyoshi, Osaka 558-8585, e-mail:

kitajima@em.elec.eng.osaka-cu.ac.jp; minami@elec.eng.osaka-cu.ac.jp

** Osaka Fuse Co. Ltd., Izumiotu, 595-0035, e-mail: koichi@fuse.co.jp stable low temperature, therefore the idea of the new fuse applied these principles for the safety. In stead, Titanium Hydride requires almost $600{ }^{\circ} \mathrm{C}$ to absorb Hydrogen into the Titanium. The method to coat the Titanium Hydride on the low melting temperature copper fuse has not been developed yet. By coating the Titanium Hydride on copper or other fuse materials, it is expected that the Hydrogen storaged in the Titanium is released when the arc is generated. After the arc is shut-down, the released Hydrogen is absorbed once again into the Titanium. So it can be said that the fuse with Titanium Hydride is really a safety and high-performance one, if it could be manufactured.

\section{PROPOSED PROCESS TO FORM TITANIUM HYDRIDE FILM}

Titanium has a high electrical resistance and is improper as a fuse itself. The fuse body requires a material of high conductivity such as copper. The copper is, as is mentioned above, the melting temperature is lower than the Hydrogen absorbing temperature. New idea was necessary to coat the Titanium Hydride on the copper surface.

New idea is created that Titanium powder is heated shortly enough for copper to be melt by pulsed high voltage and high current, and is accelerated by electromagnetic force (Fleming's law) in a Hydrogen atmosphere and the Titanium powder directly coats the copper fuse.

For this purpose, a new plasma gun is developed. During the acceleration phase of heated Titanium, Titanium Hydride is formed before it reaches the cupper surface. It is expected that the Titanium Hydride could be coated on the surface without melting the copper. 
As a method of fabrication of new-type fuse, it is thought to place the Titanium powder in a vacuum chamber and a plume of gas is injected through a valve by pulse current, then a pulsed high voltage is applied between the two electrodes as shown in Figure 1. The devices of Figure 1 is set in a vacuum chamber. The Titanium powder is accelerated by electromagnetic force due to Fleming's law. The melted Titanium powder is hit by the copper thin film of $18 \mu \mathrm{m}$ in thickness.

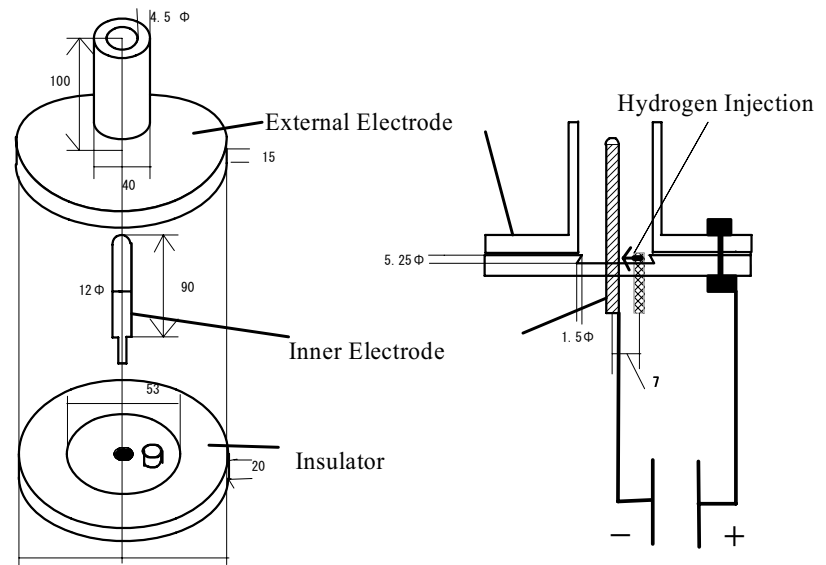

Fig. 1 An illustration of the powder injector

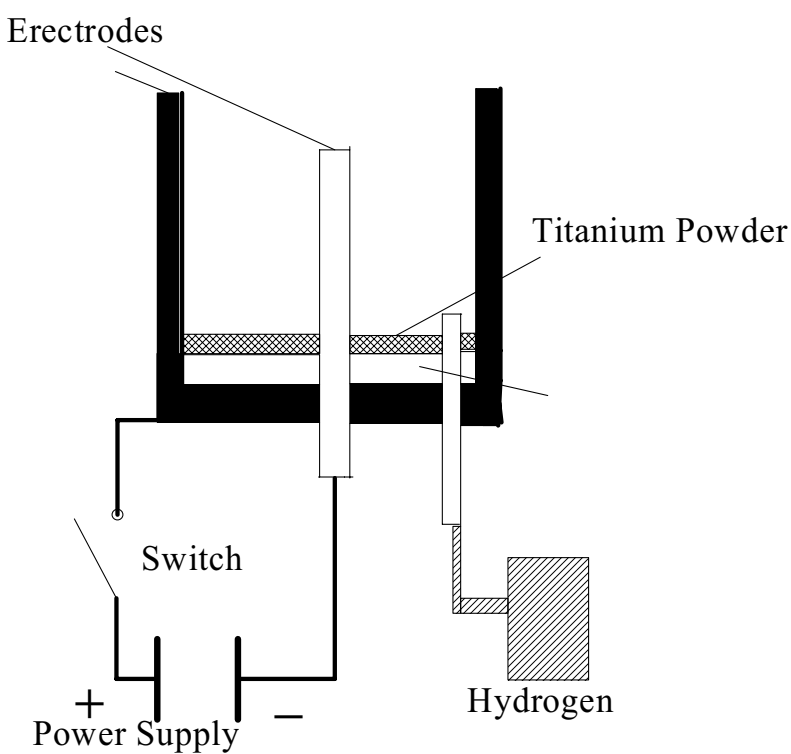

Fig. 2 The experimental set for the production of fuse

\section{EXPERIMENT RESULT}

Figure 2 shows the experimental setup. The inner electrode and the outer electrode are made of Titanium. The Hydrogen is injected into the chamber by a fast acting solenoid valve connected between the Hydrogen gas tank and the vacuum chamber. When triggered, the magnetic valve is opened and the activated Hydrogen is injected into the vacuum chamber.

The fundamental structure of thin film production used for this experiment is shown in Figure 3. The co-axial electrodes for the discharge are shown. At first, Copper film coated on a ceramic film is set in the vacuum chamber. Titanium powder is also set as shown in Figure 1. The injected gas pushes the powder upward as shown in Figure 4. Discharge is made after the Hydrogen gas is injected. The heated and melted Titanium is accelerated by the force of Fleming's law and reaches the plate. The Titanium Hydride film is coated on the copper fuse as shown in Figure 5. The equation of Fleming's Law which indicates the direction of force applied to an electric current by a magnetic field which is perpendicular to the current; and the direction of an induced force due to the Fleming's law generated in a conductor placed perpendicular to a magnetic field, when that conductor is moved perpendicular to the magnetic field.

The electrodes are coaxial, separated by an insulating plate and connected to the pulse power supply circuit. A solenoid valve is attached to the bottom of the insulating plate and injects activated gas between the electrodes. This swirls the powder up between the electrodes.

Figure 8 shows the electron microscope image of cross section of the copper film. The image shows that the thickness of the coated Titanium is $5 \mu \mathrm{m}$. The applied voltage to produce the fuse is $3.5 \mathrm{kV}$ and the size of the fuse is $4 \mathrm{~mm} \times 40 \mathrm{~mm}$. According to the calculation, the weight of one fuse is $3.2 \times 10-3 \mathrm{~g}$. It is known that $2 \mathrm{~g}$ of Hydrogen can be stored in $48 \mathrm{~g}$ of Titanium Hydride. It means that volume of stored Hydrogen of each fuse is $1.5 \mathrm{cc}$. By forming a film of Titanium Hydride on the copper film, a high performance fuse of short arc generation time is made as shown in Figure 8 and 9. A fuse made without Titanium Hydride in Figure 4 shows that the arc breaking time is $50 \mathrm{~ms}$ (Figure 8), but it is improved to be approximately $1 \mathrm{~ms}$ for the fuse with Titanium Hydride as shown in Figure 9.

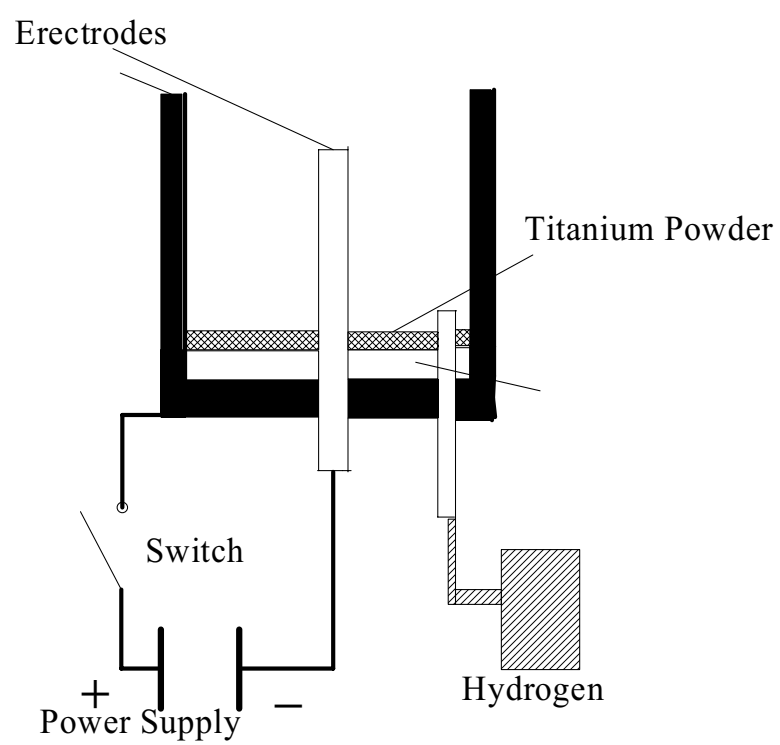

Fig. 3 The experimental set up of the experiment 


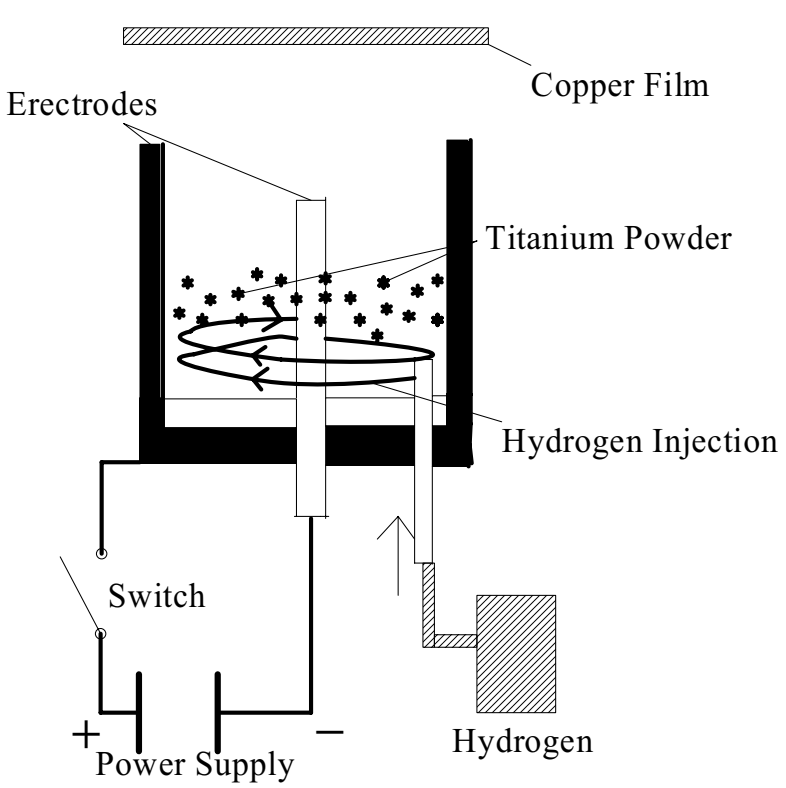

Fig. 4 The image of the ignition mechanism of the discharging

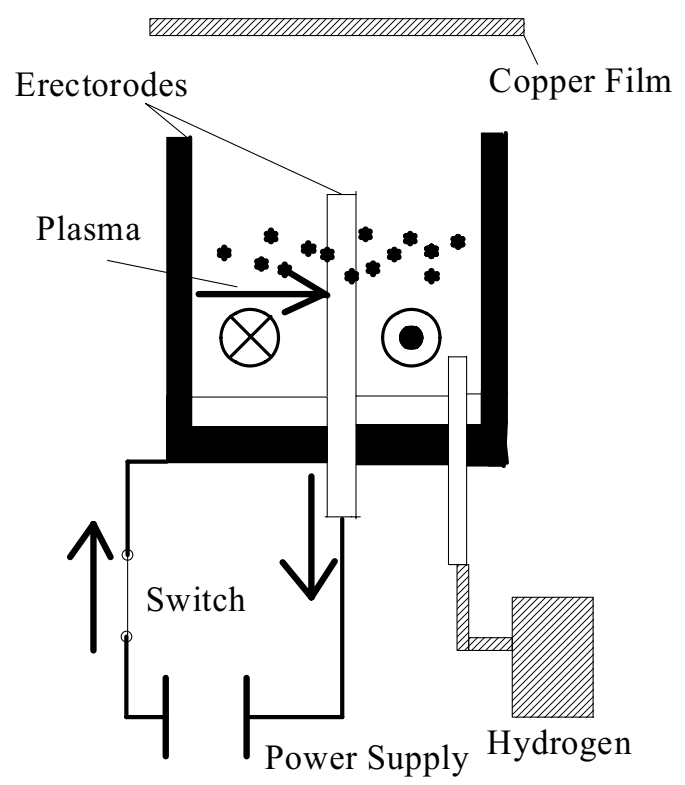

Fig. 5 The mechanism of plasma acceleration due to Fleming's law. The induced magnetic field is shown as circles. The direction of the current (about $10 \mathrm{kA}$ ) is also shown as arrow.

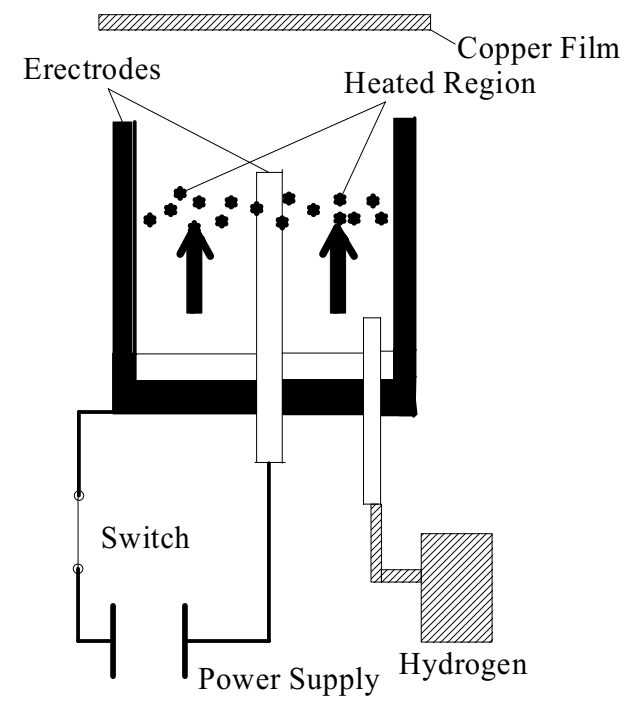

Fig. 6 The image of the accelerated Hydrogen plasma with the powder. The accelerated and melted Titanium absorbs Hydrogen and is deposited on the copper film.

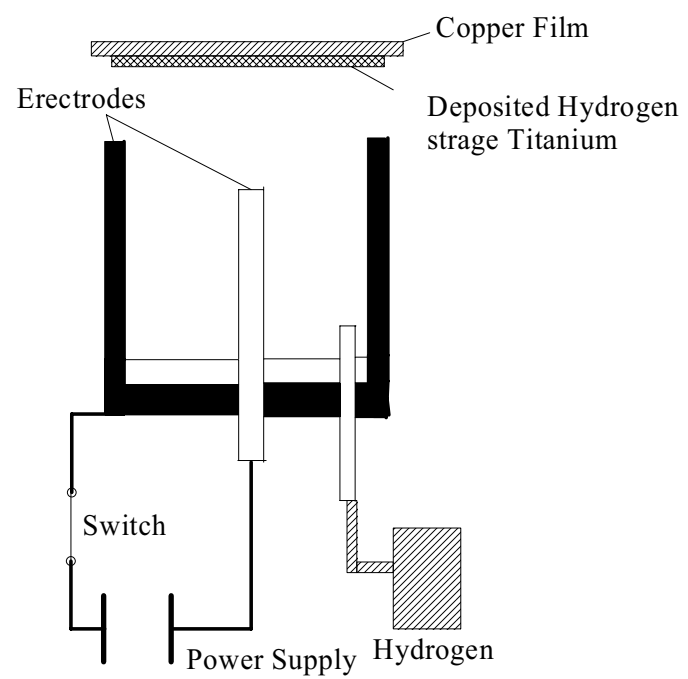

Fig. 7 The process of deposition of the Titanium Hydride

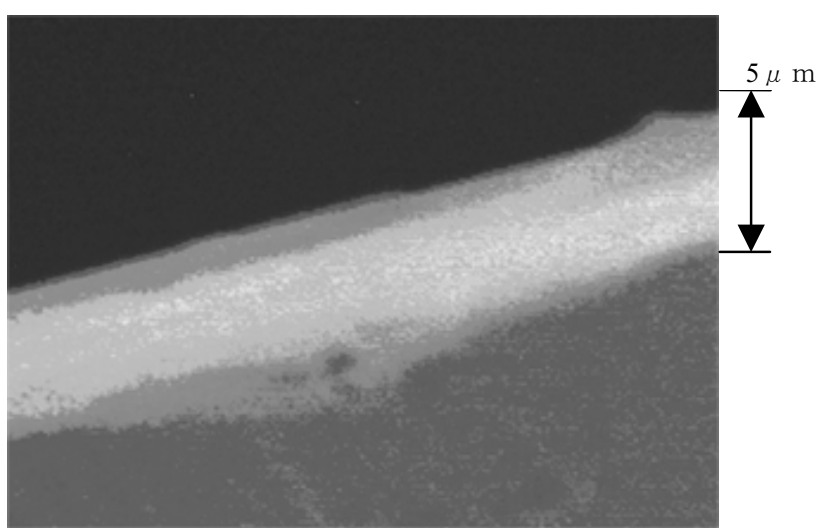

Fig. 8 A cross-sectional image of the Titanium Hydride on the copper film 


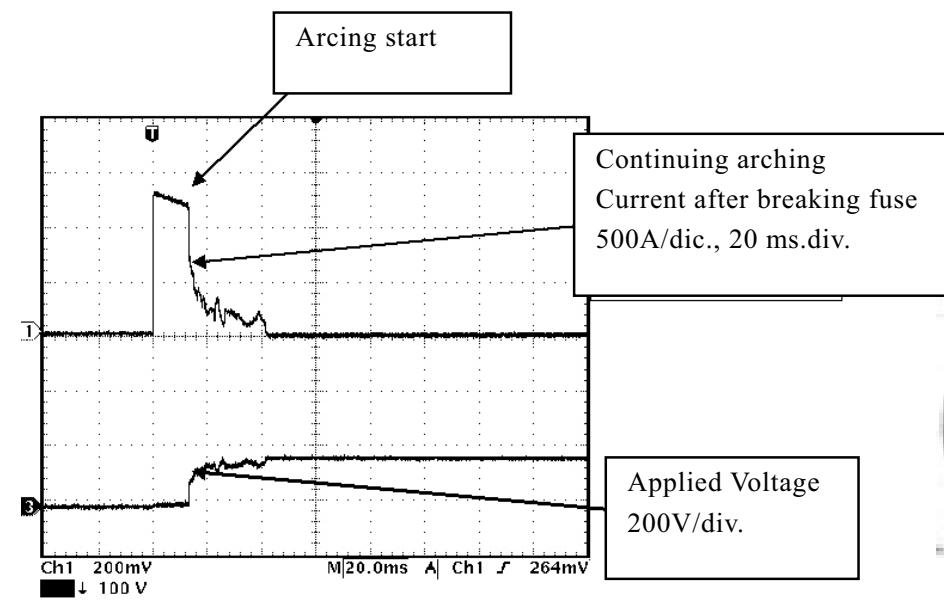

Fig. 9 An example of non Titanium coated fuse characteristics during the fuse breaking

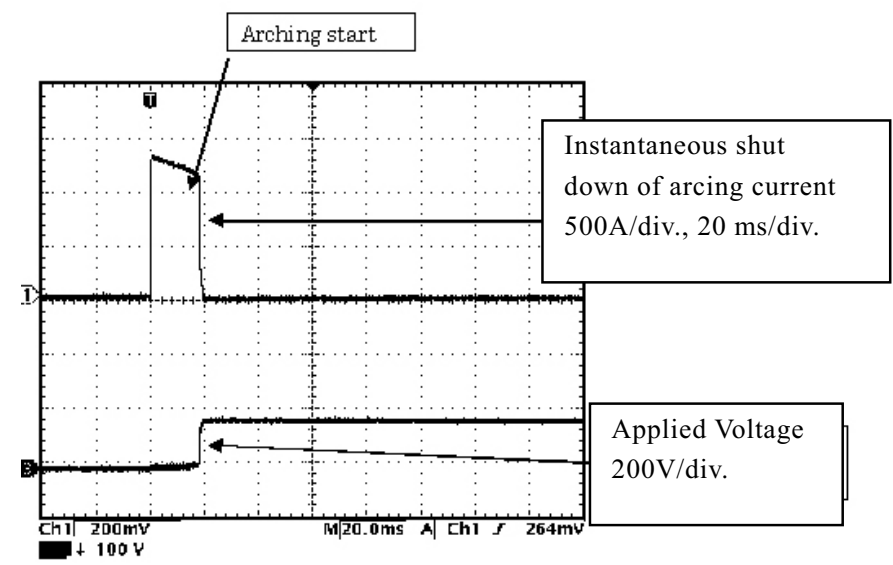

Fig. 10 An example of newly developed fuse characteristics during the fuse breaking

\section{DISCUSSION AND CONCLUSION}

The new process of developing high performance electric fuse and its feature is desicribed. This newly developed device can accelerateTitanium powder electromagnetically and a thin film of Titanium Hydride can be coated Titanium on a copper substrate using Hydrogen gas discharge. When this thin film is deposited on the surface of a copper fuse, it is shown that the Hydrogen gas emitted from the hydride makes it possible to minimize the arc time. It is explained that the negative ions of Hydrogen stop arcs, hence making a fuse that blows at a high speed. In fact, a prototype fuse exhibited a breaking time of $1 \mathrm{~ms}$. The production cost of this method seems to be lower than the previous method using low temperature Hydrogen storage metal powder contained in a cylindrical fuse [Minami et al, 2003]. Technical issue toward the next step is to improve the effency of manufacturing. It is expected that this fuse will spread on applications with electric vehicles as well as other high voltage power supply.

\section{REFERENCES}

[1] Minami S., Y. Kudose, and K. Matsumoto, Y. Takakuwa: "Newly Designed Electric Fuse for High-Power DC Power Supply", ITE Letters, 6, pp. 256-259, 2003.

\section{BIOGRAPHIES}

Miki Kitajima,

Doctoral course student, Department of Electrical Engineering, Osaka City University

Research field: Development of electric fuse for high-power application, kitajima@em.elec.eng.osaka-cu.ac.jp

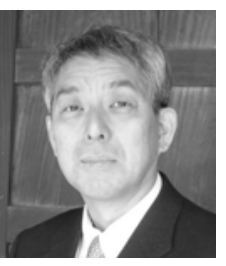

Shigeyuki Minami

Professor, Department of Electrical Engineering, Osaka City University Research field: Electromagnetism, Space Plasma Physics, Electric

Vehicles

General Secretary of Asian Electric Vehicle Society minami@elec.eng.osaka-cu.ac.jp

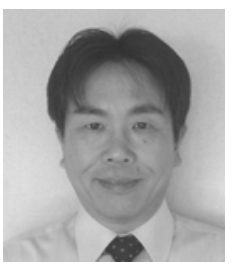

Koichi Matsumoto

Executive Director, Osaka Fuse Co., Ltd.

Research Field: Electric Fuse for Power Application koichi@fuse.co.jp 